underwent to percutaneous vertebroplasty while $84.6 \% \pm 17.1 \%$ had spinal orthoses. Pharmacological treatment for pain was prescibed to $98.2+7.1 \%$ of subjects: acetaminophen (42\%), tapentadol (24\%), opioids (24\%), NSAID (6\%) and codeine with acetaminophen (4\%). In $95 \%$ of patients with spinal orthoses drugs for pain were assumed. In about $40 \%$ of cases NSAID was switched to acetaminophen, in $18 \%$ opioid and tapentadol switched to NSAID or acetaminophen. Only a few titration of opioids/tapentadol were reported. Not adequate pain relief (NRS scale 6.2 \pm 3.1 ; QUALEFFO-41 pain score $70 \pm 14.2$ ) and impairment quality of life (mean total QUALEFFO-41 score 65.1 \pm 20.1 ) were reported.

Conclusions: With the limits of the study design and low number of cases, preliminary data seem to confirm an inadequate pain relief in PVF. The emerging critical issues crossing all cathegories of physicians are the lag of diagnosis, the inappropiate use of acetaminophen, the missing tritation of opioids or tapentadol. A definition of optimal management of acute vertebral fracture is missing due to conflicting and scarce evidences in this field predisposing to chronic pain and disability.

Disclosure of Interest: None declared

DOI: 10.1136/annrheumdis-2018-eular.5427

\section{AB0994 IMPACT ON THE ADHERENCE AND PERSISTENCE OF DENOSUMAB VS WEEKLY BISPHOSPHONATE IN HEALTH-RELATED QUALITY OF LIFE IN POSTMENOPAUSAL OSTEOPOROSIS}

G. Carlino ${ }^{1}$, R. Cinzia ${ }^{1}$, R. Maggio ${ }^{2} .{ }^{1}$ Rheumatology, ASL LE, Casarano; ${ }^{2}$ Internal Medicine, "Veris Delli Ponti" Hospital, Scorrano, Italy

Background: Long term adherence and persistence in patients undergoing treatment for postmenopausal osteoporosis remains poor despite the proven efficacy of the therapy.

Objectives: In this study, we evaluated whether greater adherence and persistence in treatment can lead to an improvement in the quality of life.

Methods: A cohort of 268 patients, all women, in postmenopausal osteoporosis divided into two groups was evaluated: "DEN Group" (DEN) in treatment with denosumab ( $n=131)$ and "BIS Group" (BIS) in treatment with bisphosphonates $(n=137)$. Table 1 shows demographic and clinical data. Patients were followed for 3 years with baseline, 6 month, 18 month, and 36 month evaluation. The evaluation criteria were the persistence in therapy and the self-related treatment compliance, as well as the quality of life assessed with the 41-item Quality of Life questionnaire for osteoporosis (QUALEFFO-41) performed at baseline, at 18 and at 36 months.

Results: Table 2 shows the percentage of patients who abandoned treatment at different times with a statistical significance towards both 18 and 36 months. In the BIS the main reason for abandonment were the adverse events (gastrointestinal, dental interventions, etc.), in the DEN the abandonment was due to drop-out. [In BIS, the most frequent reason for non-compliance with therapy was oversight, and most patients who continued treatment always used the drugs regularly on the recommended days and dosages.] In the period of time considered the majority of patients both BIS and DEN said they were satisfied with the treatment and wanted to continue it. The QUALEFFO scores (Fig 1) of patients from the baseline visit were significantly improved in the 36 month visit (BIS $79.6 \pm 25.4$ vs 65.4 \pm 14.6 ; DEN $80.2 \pm 18.5$ vs $55.6^{*} \pm 16.4\left({ }^{*} \mathrm{p}<0.001\right)$; the difference was not significant between BIS and DEN groups at both baseline visit and 36 month visit, but in the DEN group there was significance between baseline and 36 month visit.

Abstract AB0994 - Table 1. Baseline characteristics (Mean \pm SD)

$\mathrm{OC}=$ Osteocalcin, $\mathrm{CTX}=\mathrm{C}$ terminal telopeptide, $\mathrm{PTH}=$ parathyroid hormone,

25OHD=25 hydroxyvitamin D;

Abstract AB0994 - Table 2. Percentage of patients who abandoned.

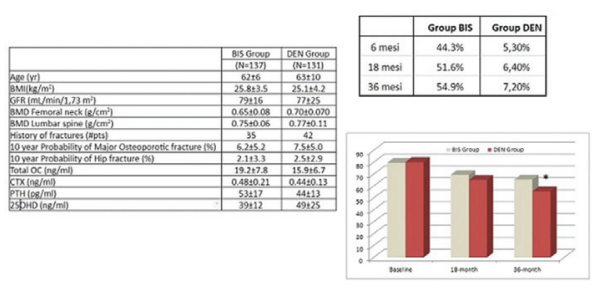

Abstract AB0994 - Figure 1. Qualeffo-41 Score $\left({ }^{*}\right.$ see text)
Conclusions: In conclusion, the observation, although numerically limited, notes that the use of denosumab in patients with postmenopausal osteoporosis leads to a greater persistence in treatment and a statistically significant adherence to therapy, which allows to obtain the maximum therapeutic effect of the therapy, also determining in 36 months of treatment an improvement in the quality of life, which is not achieved in subjects treated with bisphosphonates.

Disclosure of Interest: None declared

DOI: 10.1136/annrheumdis-2018-eular.3982

\section{AB0995 VERTEBRAL FRACTURES CASCADE: POTENTIAL ETIOLOGIES AND RISK FACTORS}

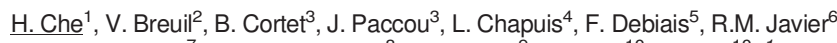
N. Mehsen Cetre ${ }^{7}$, S. Loiseau Peres ${ }^{8}$, T. Thomas ${ }^{9}$, C. Roux ${ }^{10}, \mathrm{~K}_{\text {B Briot }}{ }^{10} .{ }^{1} \mathrm{CHU}$ Lapeyronie, Montpellier, ${ }^{2} \mathrm{CHU}$ Nice, Nice; ${ }^{3} \mathrm{CHU}$ Lille, Lille; ${ }^{4} \mathrm{CH}$ du Vitre, Vitre;

${ }^{5} \mathrm{CHU}$ Poitiers, Poitiers; ${ }^{6} \mathrm{CHU}$ Hautepierre, Strasbourg; ${ }^{7} \mathrm{CHU}$ Bordeaux, Bordeaux; ${ }^{8} \mathrm{CHR}$ Orleans, Orleans; ${ }^{9} \mathrm{CHU}$ Saint Etienne, Saint Etienne; ${ }^{10} \mathrm{CHU}$ Cochin, Paris, France

Background: Vertebral fracture (VF) is the most common osteoporotic fracture, and a strong risk factor of subsequent vertebral fracture. Prospective studies have shown that a recent VF increases an imminent risk of a subsequent one, and attention has been paid recently to a possible cascade phenomenon i.e. the occurrence of multiples VFs in less than one year.

Objectives: This cascade could have severe consequences, and we prompted a study to identify potential causes of osteoporosis and risk factors.

Methods: Vertebral fractures cascade (VFC) observations were collected retrospectively between January 2016 and April 2017. VFC was defined as the occurrence of at least 3 vertebral fractures within one year. Patients with othe etiologies than osteoporosis (i.e. malignant or traumatic VFs) were excluded. The cause of osteoporosis associated with VFC was the one retained by the physician at the time of diagnosis.

Results: Ninety-five observations of VFC ( $80 \%$ of women, mean age of 71 years) were collected in 10 centres ( 9 tertiary centres and 1 outpatient centre). The median number of incident VFs over 1 year was $4 .^{3-11}$ Forty-five patients $(45.9 \%)$ had a previous major fracture before the VFC and $65(70.7 \%)$ had densitometric osteoporosis (T-Score $\leq-2.5$ SD either at lumbar or femoral site). Eighteen (19\%) patients currently received oral glucocorticoids treatment at the time of VFC, with a mean daily dose of $20 \mathrm{mg}$. Thirty-three (35.1\%) patients received systemic glucocorticoids in the past. The main comorbidities were history of cancer $(n=19)$ and chronic inflammatory diseases $(n=21)$ including asthma $(n=7)$, chronic obstructive pulmonary disease $(n=7)$ and rheumatoid arthritis $(n=7)$.

A secondary osteoporosis associated with the cascade was diagnosed in 54 patients $(54.5 \%)$ with the following causes: glucocorticoid-induced osteoporosis $(n=22,23.7 \%$ ), benign hemopathies (mastocytosis, MGUS) $(n=7,7.1 \%)$, use of aromatase inhibitors $(n=3,3.1 \%)$, anorexia nervosa $(n=3,3.1 \%)$, alcoholism $(n=3$, $3.1 \%)$, pregnancy and lactation-associated osteoporosis $(n=2,2.1 \%)$, primary hyperparathyroidism $(n=2,2.1 \%)$ and hypercorticism $(n=1,1.1 \%)$. In addition, 11 cases $(11.3 \%$ ) were reported following a vertebroplasty procedure.

Primary either postmenopausal or idiopathic osteoporosis was diagnosed in 48 patients $(51.6 \%)$. A total of $29(29.6 \%)$ patients previously received an anti-osteoporotic treatment. In six patients $(6.3 \%)$, VFC occurred early (in the year) following discontinuation of an anti-osteoporotic treatment: 5 after denosumab and one 12 months after an infusion of zoledronic acid.

Conclusions: The results of this retrospective study show that almost half of VFC occurred in patients with secondary osteoporosis. While they suggest that a careful management has to be given to these patients in order to prevent VFC in these circumstances, prospective studies are needed to further explore the determinants of such a severe complication of osteoporosis.

Disclosure of Interest: None declared

DOI: 10.1136/annrheumdis-2018-eular.2543

\section{AB0996 1 BONE MINERAL DENSITY AT DIFFERENT SITES AS A PREDICTOR OF RIB FRACTURES: A CASE-CONTROL STUDY}

H.L. Wu, M. Bukhari. Rheumatology, University Hospitals of Morecambe Bay NHS Foundation Trust, Lancaster, UK

Background: Rib fractures commonly occur as a result of direct trauma, though pathological causes have also been identified. Literature on the specific risk factors of rib fractures is scarce. There was an American prospective cohort study 
which highlighted the relationship between risk factors of osteoporosis and rib fractures in older men aged 65 or above. ${ }^{1}$ It was observed that the incidence of rib fractures was $3.5 / 1000$ years. Only $3 \%$ of rib fractures occurred with absence of trauma. Bone mineral density (BMD) is an important measure for predicting various bone fractures. ${ }^{2}$ However, prediction of rib fractures using BMD measurement in different body sites is not reported.

Objectives: To determine if reduction in femoral neck and lumbar spine BMD are predictive of rib fractures.

Methods: Patients referred from primary care to a DEXA scanner in the north west of England between January 2006 and December 2016 were used in this study. Patients with a history of rib fractures at first scan were matched with controls who did not have any indication for scanning. Cases and controls were matched for age and gender. Differences in BMD at L1-L4 spine and the femoral neck were analysed using two-sample t test. Logistic regression models were fitted to analyse the association between lumbar spine and femoral neck BMD and rib fracture occurrence. The fit of each model was compared using receiver operating characteristic (ROC) curves.

Results: A total of 1554 patients were included in the study ( 777 cases of rib fractures and 777 controls). Mean age for both cases and controls were 62.5 years (SD 12.0). 605 patients (77.9\%) were female in both the case cohort and the controls. The mean T score in the lumbar spine is 1.00 in cases versus 1.10 in controls (diff $0.10095 \% \mathrm{Cl} 0.0488,0.142 \mathrm{p}<0.001$ ). The mean T score in the femoral neck is 0.812 in cases versus 0.935 in controls (diff $0.12395 \% \mathrm{Cl} 0.108$, $0.137 \mathrm{p}<0.001)$. The odds of lumbar spine BMD and femoral neck BMD were 0.111 (95\% Cl 0.0640, 0.194, $\mathrm{p}<0.001)$ and 0.00209 (95\% Cl 0.000903, 0.00485, $\mathrm{p}<0.001)$ respectively. The areas under ROC curve (AUC) for lumbar spine BMD and femoral neck BMD were 0.623 and 0.733 .

Conclusions: This study demonstrated that reduction in BMD at the lumbar spine and femoral neck positively correlated to the risk of rib fractures. Reduction in femoral neck BMD is a stronger predictor of the two. Prediction of rib fractures could be affected by other factors influencing lumbar spine and femoral neck BMD. Further work in different demographic groups should be done for comparison and analysis.

\section{REFERENCES:}

[1] Barrett-Connor, et al. Epidemiology of rib fractures in older men: Osteoporotic Fractures in Men (MrOS) prospective cohort study. BMJ 2010; 340: c1069

[2] Dobrzynska, et al. Predictors of fragility humeral fractures: An Observational Study. Ann Rheum Dis 2015; 74(Suppl2):763

Disclosure of Interest: None declared

DOI: 10.1136/annrheumdis-2018-eular.1360

\section{AB0997 CLINICAL CHARACTERISTICS OF NON-RESPONDER TO DENOSUMAB TREATMENT FOR POSTMENOPAUSAL OSTEOPOROSIS IN JAPANESE WOMAN}

I. Yoshii. Orthopaedics, YOSHII HOSPITAL, Shimanto City, Japan

Background: Denosumab (d-mab), an anti-receptor activator of nuclear factor kappa-B ligand (RANKL) monoclonal antibody, is now widely used in postmenopausal osteoporosis (OP) treatment. It can attain reliable increase of bone mineral density (BMD) despite any OP drugs previous used, and that can be predicted in a long period. However, sometimes we observe BMP decrease. Factor that suppresses BMD gain is still unclear.

Objectives: This study investigates non-responder to d-mab, and attempted to evaluate factors that concerns non-responder in clinical practice retrospectively. Methods: 212 postmenopausal OP patients have been treated with d-mab continuously for more than two years under vitamin $D$ supplement and calcium since June 2013. These patients were enrolled, and their clinical background (CBs) such as age at start of d-mab, past history of bone fragility fracture, alcohol habitat, current smoking, being treated for rheumatoid arthritis (RA), glucocorticoid steroid thrown, OP drug naïve, length of administration (LA), concurrence of lifestyle disease (LSD) such as diabetes mellitus, hypertension, and chronic obstructive lung disease, and number of comorbidity (N.Com), were investigated (table 1a). BMD of lumbar spine (LS), femoral neck (FN), greater trochanter (GT), and whole femur $(\mathrm{WF})$ at the start and every six months thereafter were measured. Tartrate-resistant Acid Phosphatase 5b (TRACP5b) and total dose of type-one pro-collagen - $\mathrm{N}$ pro-peptide (P1NP) at the same time of BMD measurement, and reduction ratio $(R R)$ of them compared to the first shot were calculated. Patients were divided into two groups whether BMD increased. BMD in each part at final measurement relative to that at start was compared statistically with paired T-test. CBs were statistically evaluated between the two BMD groups (Res and n-Res; Responder and non-Responder) with Mann-Whitney U-test (MWT), and then correlation between the BMD groups and factors of $\mathrm{CB}$ that had demonstrated significantly difference, and TRACP5b and P1NP was also evaluated statistically with Binary Logistic Analysis (BLR). Statistical significance was set less than $5 \%$.

Results: For all patients, BMD in LS, WF, and GT demonstrated significant increase at final measurement than at start $(\mathrm{p}<0.001)$, while FN demonstrated no significant increase. However, n-Res counted 30, 89, 46, and 44 for LS, FN, WF, and GT, respectively. LA demonstrated negative significant correlation for being $\mathrm{n}$-Res in all part, while LSD, N.Com, TRACP5b at start, and RR of TRACP5b at second shot and last shot, demonstrated significant positive correlation in LS with MWT. However, with BLR, only RR of TRACP $5 b$ at second shot demonstrated significant negative correlation with n-Res in LS (OR:0.0702, 95\% Cl:0.00510.9655 ) with 0.39 cut-off index (COI), while no other factors but LA demonstrated significant negative correlation with n-Res in WF (OR:0.9669, 95\% Cl:0.94330.9911 ) and GT (OR:0.9632, 95\% Cl:0.9391-0.9878) with 26 and 24 months COI, respectively (table $1 \mathrm{~b}$ ).

Table 1a Patient's background. Age at Start, Length Administered, Number of Chronic Comorbidities, and Bone Mineral Density, show average value and standard deviation. The other parameters show numbers.\%YAM:\% of young adult mean value. LS: lumbar spine, FN: femoral neck, WF: whole femur, GT: greater trochanter, SERM: selective oestrogen receptor modulator

Table $1 b$ Parameters that demonstrated significant difference between responder and non-responder to denosumab, and their $p$-values, and results of these param eters with binary logistic regression analysis. LS: lumbar spine, FN: femoral neck WF: whole femur, GT: greater trochanter, LSD: concurrence of lifestyle disease LA: length of administration, N.Com: number of concurrent comorbidities, TRACP5b at start; tartrate-resistant acid phosphatase 5b (TRACP5b) at start of administration, rTRACP5b@1stP: reduction ratio of TRACP5b of second shot compared to the first, rTRACP5b@LT: reduction ratio to TRACP5b of last shot compared to the first, Drug Naive: initial drug as osteoporosis treatment, PINP total dose of type-one pro-collagen $-\mathrm{N}$ - pro-peptide.

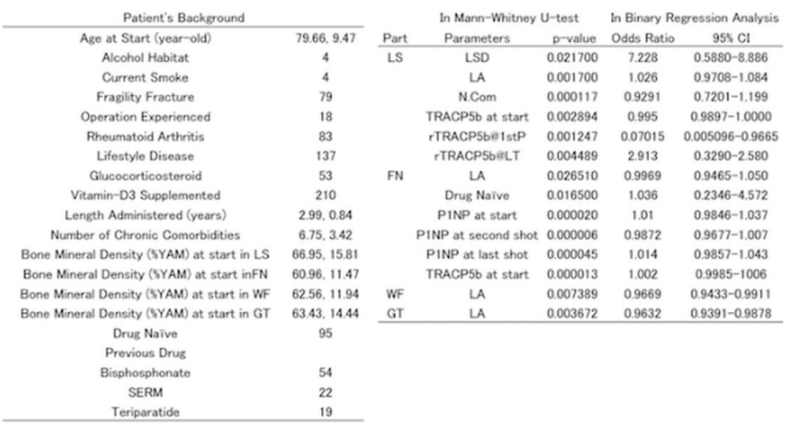

Conclusions: These results suggest that non-responder to d-mab exists. Length of administration works for BMD to increase in femur, and concurrence of LSD, and N.Com are suggested to be risk factors in LS. Less reduction ratio of TRACP $5 \mathrm{~b}$ at second shot is referred for prediction of non-responder. However, there is no parameter that predicts non-responder before administration.

Disclosure of Interest: None declared

DOI: 10.1136/annrheumdis-2018-eular.1263

\section{AB0998 OSTEOPOROSIS PREVALENCE IN POST-MENOPAUSAL PATIENTS TREATED WITH AROMATASE INHIBITORS USING BONE MINERAL DENSITY VALUES FROM A SPANISH POPULATION}

J. Loarce-Martos ${ }^{1}$, W.A. Sifuentes-Giraldo ${ }^{1}$, L.V. Maldonado-Romero ${ }^{2}$, M. AhijónLana $^{1}$, C. Velázquez-Arce ${ }^{1}$, N. Martínez-Jañez ${ }^{3}$, M. Vázquez-Díaz ${ }^{1}$.

${ }^{1}$ Rheumatology, Ramon y Cajal Universitary Hospital, Madrid, Spain;

${ }^{2}$ Rheumatology, Hospital Teodoro Maldonado Carbo, Guayaquil, Ecuador,

${ }^{3}$ Oncology, Ramon y Cajal Universitary Hospital, Madrid, Spain

Background: The majority of cases of breast cancer $(\mathrm{BC})$ are hormone receptor positive and are sensitive to antiestrogen therapy. For postmenopausal women, adjuvant hormonal therapy with an aromatase inhibitor $(\mathrm{Al})$ is the standard of care, and is associated with greater prevalence of osteoporosis (OP) ${ }^{1}$. Definition of osteoporosis is made depending on the T-Score value, which is calculated according to the bone mass peak obtained from a reference population; in our country, BMD reference values in clinical practice are obtained from the National Health and Nutrition Examination Survey cohort (NHANES III) for femoral neck (FN) and those proportionated by the commercial brand Hologic for lumbar spine (LS), which may not be representative of our population and could be distorting the assessment of OP in our patients. 